\title{
Cognitive Remediation for Schizophrenia: A Review of Recent Findings
}

\section{Felice Reddy, $P h D^{1,2, *}$ \\ William P. Horan, $P h D^{1,2}$ \\ Carol Jahshan, $P h D^{1,2}$ \\ Michael F. Green, $P h D^{1,2}$}

\author{
Address \\ ${ }^{*}$,1 VA Greater Los Angeles Healthcare System, Mental Illness Research Education \\ and Clinical Center (MIRECC) 210A, 11301 Wilshire Blvd. Bldg. 210; Room 117, \\ Los Angeles, CA 90073, USA \\ Email: lenafelice@ucla.edu \\ ${ }^{2}$ Department of Psychiatry and Biobehavioral Sciences, The Geffen School of Medicine \\ at UCLA, 11301 Wilshire Blvd., Los Angeles, CA 90073, USA
}

Published online: 9 March 2014

(C) Springer International Publishing AG (outside the USA) 2014

Keywords Schizophrenia - Cognitive remediation - CR · Neurocognition - High-level cognitive processes • Neuroplasticity · Treatment

\section{Opinion statement}

Schizophrenia and related psychotic disorders are characterized by deficits in neurocognition. Deficits in domains such as attention, memory, executive functions, and speed of processing, as well as early perceptual processes, typically appear early in the course of the disorder and remain stable over time. Cognitive deficits can cause serious impairments in community functioning (i.e., work, independent living, and social relationships). For this reason, cognitive remediation (CR) is increasingly utilized to improve neurocognition. The empirical support for CR for adults with chronic schizophrenia is encouraging and growing. CR approaches are suitable for widespread dissemination and have been the focus of the majority of recent empirical research in psychoses. There are two broad categories of computerized CR approaches: those that target higher-level cognitive processes, and those that target neuroplasticity using basic auditory and visual processing. Research currently supports the efficacy of both higher-level and neuroplasticity-based CR for improving targeted cognitive domains; however, there is more consistent support for higher-level approaches in terms of generalization to untrained cognitive domains and functional outcomes. To achieve functional outcomes, CR combined with skills training or other psychosocial rehabilitation approaches is likely the most effective approach. The majority of CR interventions allow difficulty to be individually adjusted, which is an important therapeutic feature, and some provide an array of modules to allow personalized interventions. Several CR interventions appear to have durable treatment benefits, but more research is needed to 
clarify whether booster sessions, pharmacological augmentation, or other treatments should routinely be incorporated into treatment plans.

\section{Introduction}

Neurocognitive impairments are core features of schizophrenia [1, 2]. Patients show large (standardized effect size $>1.0$ ) deficits across the cognitive domains of attention, learning and memory, working memory, speed of processing, and reasoning and problem solving (i.e., executive functions), as well as in basic auditory and visual perceptual processes [3-5]. These deficits are evident in the prodromal phase, tend to worsen around first episode, and subsequently remain stable, typically persisting in the residual phase and after successful amelioration of positive symptoms [6•]. These impairments are also strongly linked to poor functional outcomes across the domains of independent living, work/education, and social relationships. Partly because of their role in determining level of community functioning, there has been an interest over the past 25 years to develop training methods to remediate neurocognitive deficits.

Cognitive remediation (CR) refers to interventions that are based on learning principles that target cognitive deficits and aim to generalize to improvements in functional outcomes (Cognitive Remediation Experts Workshop; CREW, 2010). CR modalities have been developed and evaluated in over 45 independent randomized controlled trials in schizophrenia. Among the various $C R$ approaches, a broad distinction can be made between those focused on higher-level cognition versus neuroplasticity-oriented approaches. Higher-level CR approaches, which directly target cognitive domains such as attention, memory and executive functions, have received the lion's share of research attention. A relatively recent advance in CR over the past 5-10 years is the emer- gence of neuroplasticity-oriented interventions, which were hoped to be a more effective, neuroscience-based approach to CR. Neuroplasticity refers to the changes in cortical organization and interaction between brain systems in response to learning [7]. Neuroplasticity-based approaches rest on the premise that repetitive high-dosage training in basic perceptual and lower-level cognitive operations facilitates synaptogenesis, which leads to changes in higher-order cognitive functions [8]. Both higher-level and neuroplasticity-based interventions can be administered as stand-alone interventions, or along with additional psychosocial interventions such as skills-training or bridging groups to help the newly acquired cognitive abilities to generalize.

The extensive body of research on CR for schizophrenia has generally demonstrated the promise of improving cognition through training approaches. Indeed, a large meta-analysis in 2011 concluded that CR has durable effects on global cognition and functioning, and that these effects appear to be stronger when CR is combined with psychiatric rehabilitation [9•]. Since the publication of that meta-analysis, trends in treatment and assessment highlight some important updates to our current understanding of CR effectiveness in schizophrenia, and also leave several unanswered questions. In the following sections, we will discuss the firm conclusions we can draw from the recent (past 3 years) empirical trials on higher-level and neuroplasticity-based CR for psychotic disorders. We will then discuss the new research and treatment directions, unanswered questions, and promising future directions.

\section{Higher-level CR approaches}

Our review of the higher-level studies published between 2011 and 2013 (see Table 1) includes 16 peer-reviewed studies of CR for schizophrenia. The majority of studies in this review were randomized, controlled trials. The patient sample sizes range from 17 to 107 with a mean of 63; three studies included a healthy control sample. Two studies also included follow-up assessments, at least 1-year post intervention, to examine durability of treat- 


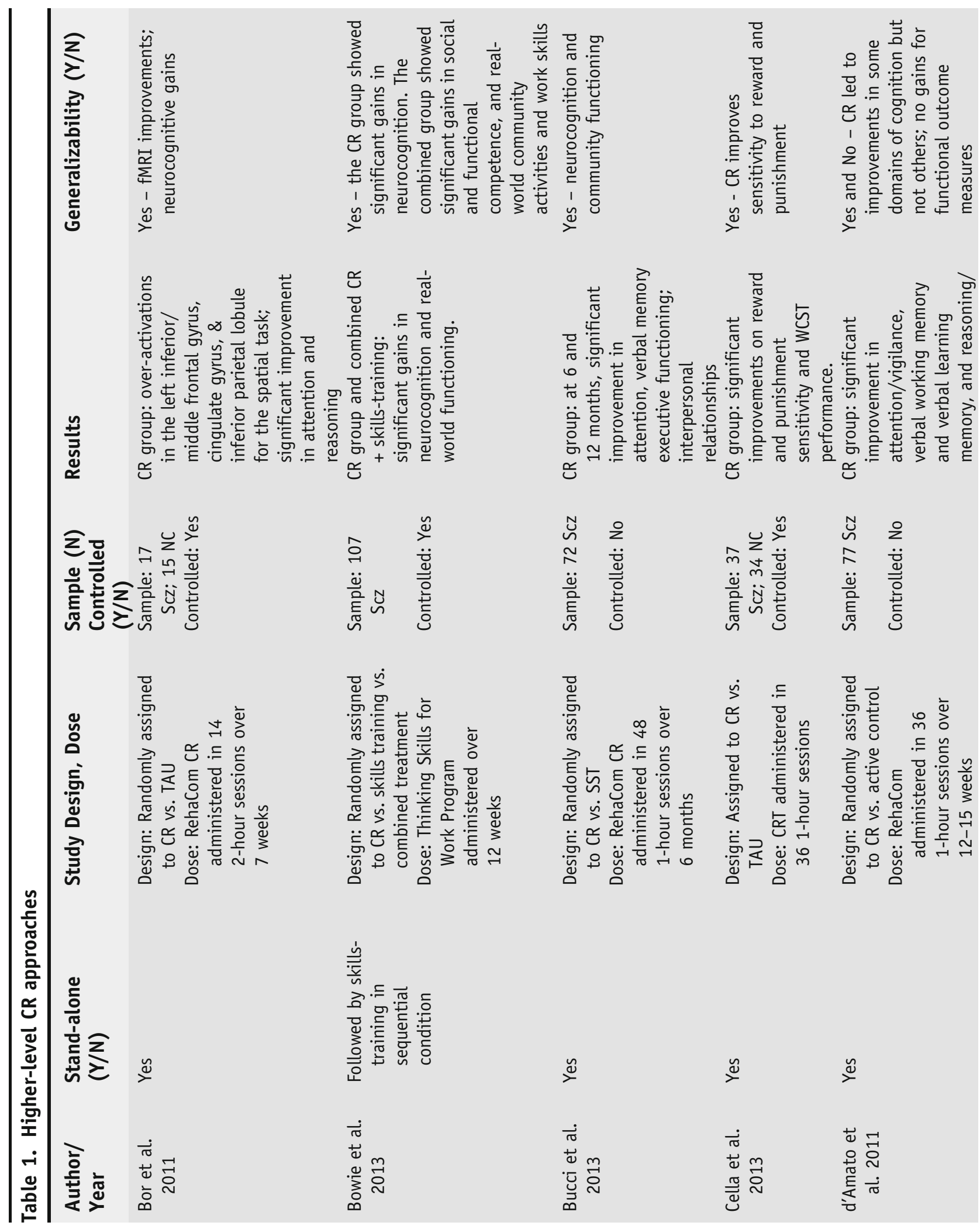




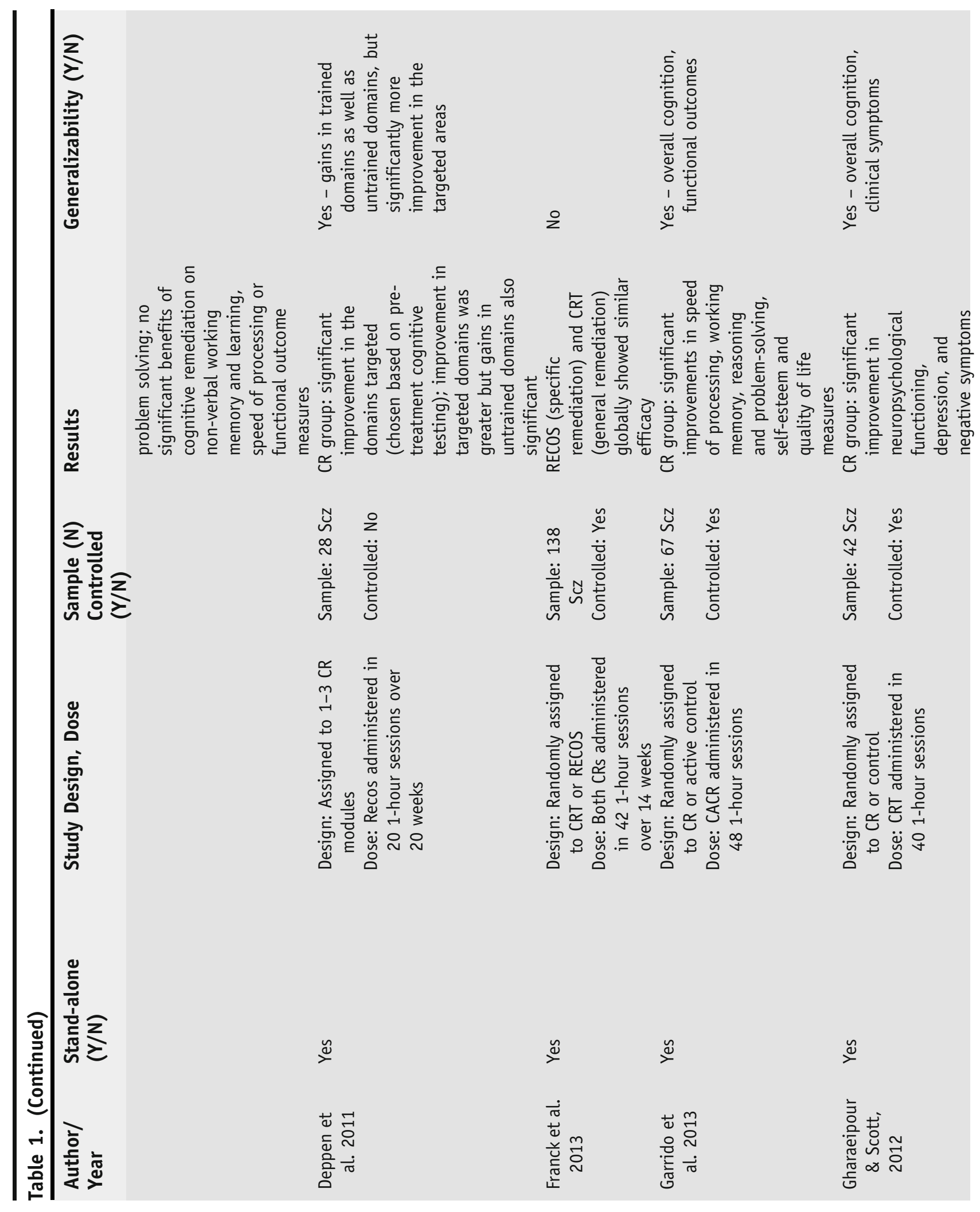




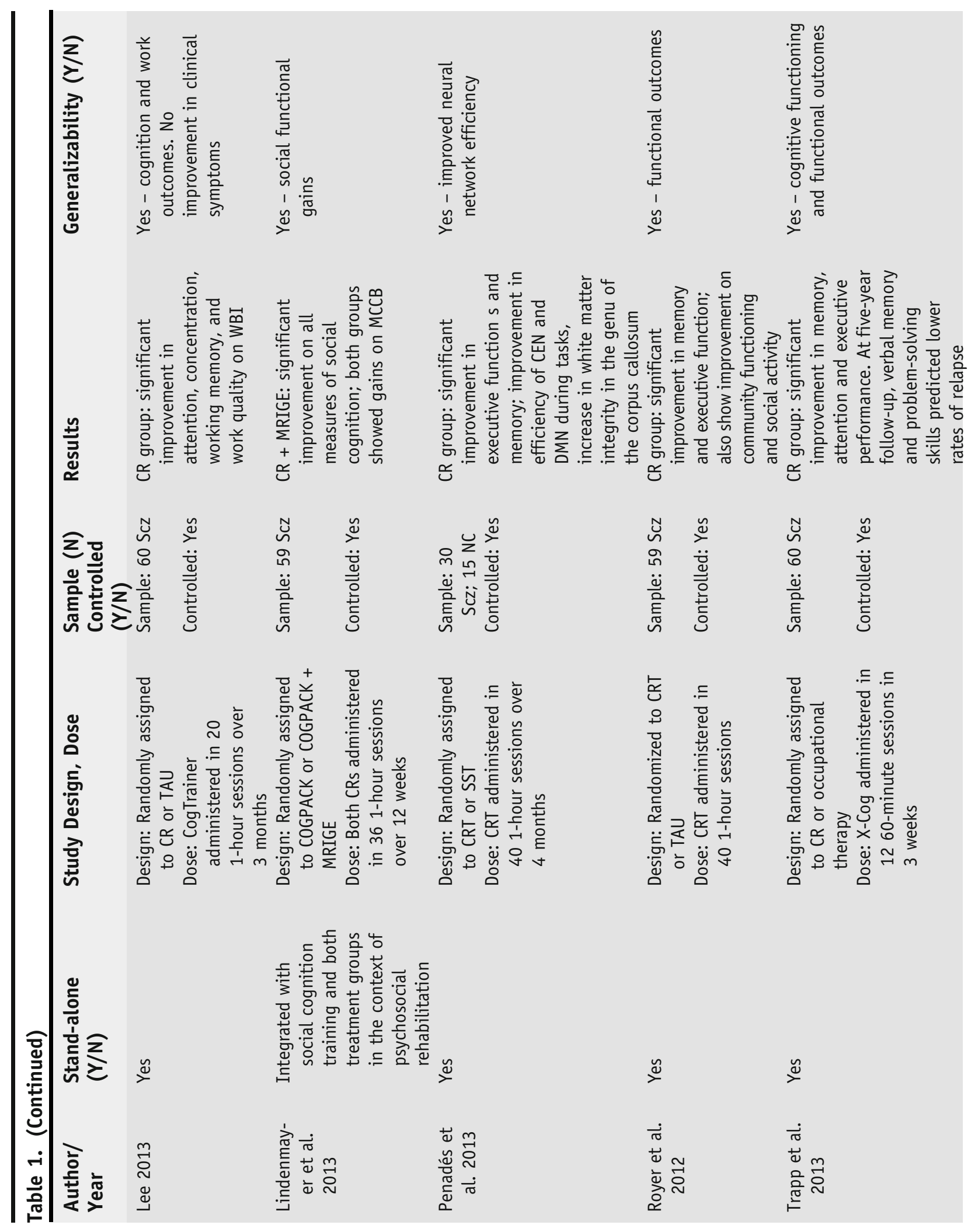




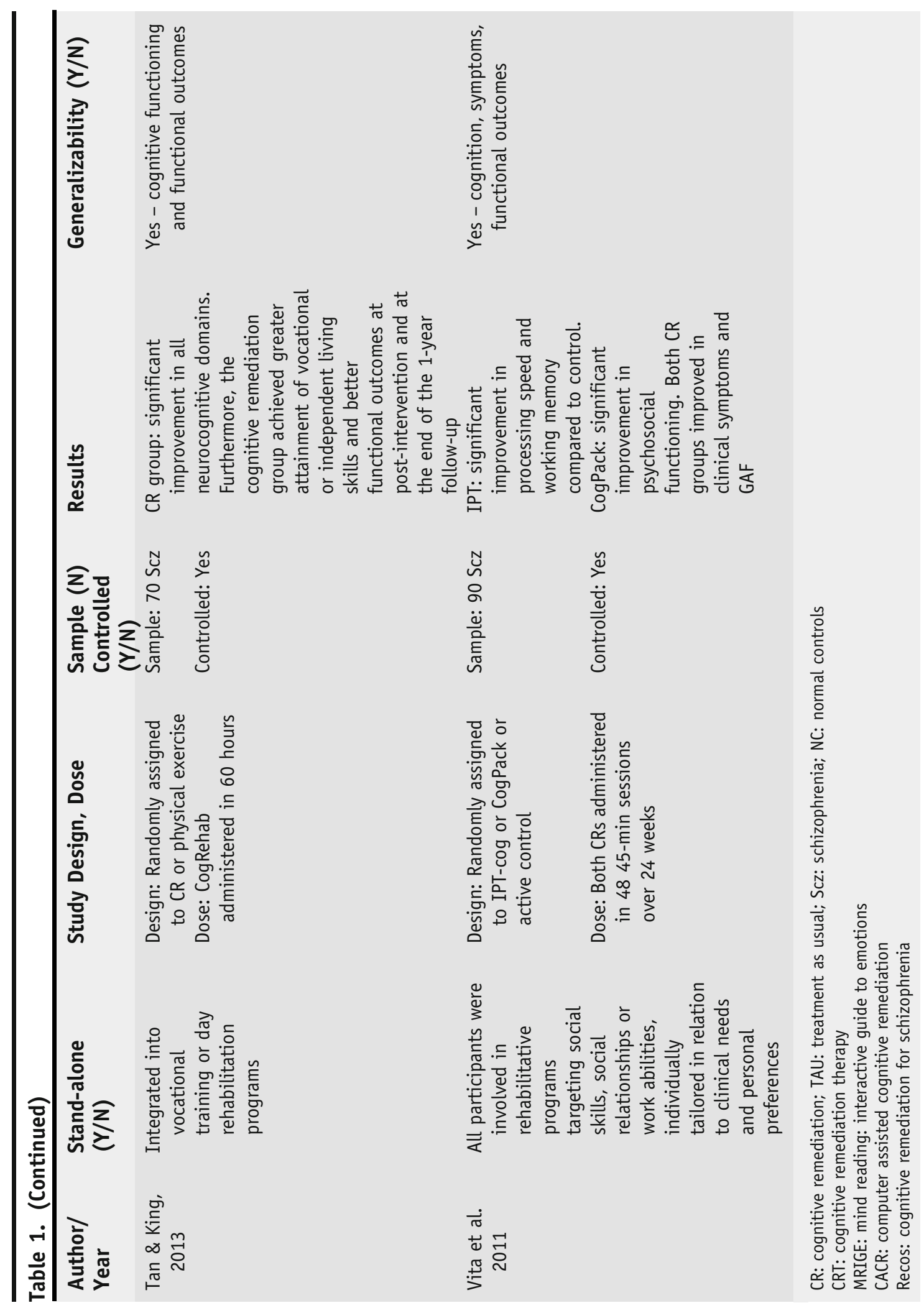


ment effects. The general impression is that many research laboratories are examining the efficacy and effectiveness of CR for psychotic disorders. We included only studies that looked at cognition as the primary outcome, although some studies implemented CR to target social cognition or another related functional ability. There are three salient issues across all recent CR studies: first, whether treatment gains generalize across domains of cognition and to functional outcomes; second, the extent to which CR gains impact neural structures and functions; and third, determination of a "best" methodology for treatment delivery.

First, the studies support the notion that improvements in specific targeted cognitive domains generalize to other untrained domains. All sixteen studies reported gains in several cognitive domains, and in general cognition. One study found gains in all cognitive domains, but significantly greater ones in trained domains [10]. Another study compared higher-level training that targets specific deficit areas (Recos) to general higher-level CR training and found both groups improved on executive functioning, and there was no evidence to support either a specific or general approach to higher-level CR [11]. Regarding generalization to functional outcomes, the recent research also indicates that gains tend to generalize to real-world skills. Ten of the sixteen studies found supportive evidence of generalizability to functional outcomes [12-21]. One study [22] reported no improvement in functional outcome and another study [12] found that functional outcomes improved when skills-training followed CR, but not after CR alone. Although it is not a focus of this review, a few studies observed improvements in clinical symptoms after $\mathrm{CR}$ and posit that neurocognitive impairment is closely associated with negative symptoms [23]. Thus, the conclusion across studies is that higher-level CR results in improved neurocognition across domains, and generalizes to work, social, and independent living skills.

A recent trend is the examination of CR impact on neural functioning. There is some evidence that higher-level CR training impacts neural functioning. Two of the 16 studies in the table $[24,25]$ used magnetic resonance imaging (MRI) to assess white matter integrity and functional MRI (fMRI) to measure regional brain activity of relevant brain regions; both showed positive results. In studies of higher-level computerized CR not included in this review because of publication date, repeated structural MRI assessments show CR resulted in significantly greater preservation of gray matter volume [26] and increased activation in attention and working memory networks (dorsolateral prefrontal cortex, anterior cingulate, and the frontal pole) [27]. Furthermore, these preservations mediated the effects of the CR to predict better cognitive outcomes. However, few studies have examined such effects and larger studies are needed for clarification of the structural and functional changes.

An ongoing debate in this area is whether there is an optimal approach to delivering CR. The primary methods include drill and practice (either administered by a trained therapist or via computerized modules), and drill and practice combined with adjunctive psychiatric rehabilitation. The recent research does not resolve this issue, as gains are apparent in studies with and without adjunctive treatments. Only four of the recent 16 studies reported systematic integration of higher-level approaches with structured approaches for psychosocial treatment $[12,17,20,21]$. These studies all yielded positive 
results for functional outcomes, including work outcomes and social skills. Six of the 12 studies that did not implement adjunctive psychosocial rehabilitation treatment also showed generalization to functional outcomes. Previous meta-analyses found more robust gains for integrated treatments [28], and our current review does not support one side of this argument.

\section{Neuroplasticity-based approaches}

As summarized in Table 2, we identified five neuroplasticity-oriented intervention studies published between 2011 and 2013. There were significantly fewer studies of neuroplasticity-based CR than there were studies of higherlevel approaches. Three of the five studies were controlled trials with random assignment and one was a multi-site trial [29]. The patient sample sizes ranged from 14 to 55 with a mean of 41 . One of the five studies included a healthy control sample and one of the five studies was primarily seeking to establish feasibility. Two of the studies included electrophysiological measurement of treatment effects. Two of the studies included adjunctive psychosocial treatment, and one study directly compared neuroplasticity-based with a higher-level approach. Again, we included only studies that looked at cognition as the primary outcome. We review the same three salient issues in relation to recent neuroplasticity-based CR that we examined above.

First, we examined the evidence with regard to generalizability to untrained cognitive domains and functional outcomes. The five recent studies all found that neuroplasticity-based interventions do not generalize across domains of cognition. Of the five studies we reviewed, two observed definite gains on tasks related to training exercises $[30 \bullet 31]$, one study reported trend-level improvements on overall cognition [29], and one study observed gains in the three areas of cognition that were assessed (immediate and delayed recall and working memory) [32]. One study assessed generalization to functional outcomes, but did not find support [30]. Overall, this is a small sample of studies and the negative findings are in contrast to two studies with positive results outside of our time period $[33,34]$ that found evidence of generalizability to neurocognition and functional outcomes (i.e., work skills).

Regarding the question of whether CR has a detectable impact on brain functioning, two of the five studies measured changes at the neural level. One reported significant effects, in terms of normalized magnetoencephalography (MEG) sensory gating (M50 ratio) following neuroplasticity-based CR training [32]; in a separately published re-examination of the data, the researchers found normalized alpha desynchronization mediated the effects of improved auditory gating [35]. The other study concluded there were no gains in neurocognition and no changes in electroencephalogram (EEG) measures following 40 hours of BrainFitness training [36]. Two other studies $[37,38]$ that were published before our publication period showed improved verbal memory and auditory neural responses (M100) after intensive auditory training. The MEG findings contradict Rass et al.'s study [36], but are in line with the original neuroplasticity-based studies from Fisher and colleagues [34, 39], which reported significant improvements in verbal learning and memory and global cognition, and maintenance of treatment gains at 6- 


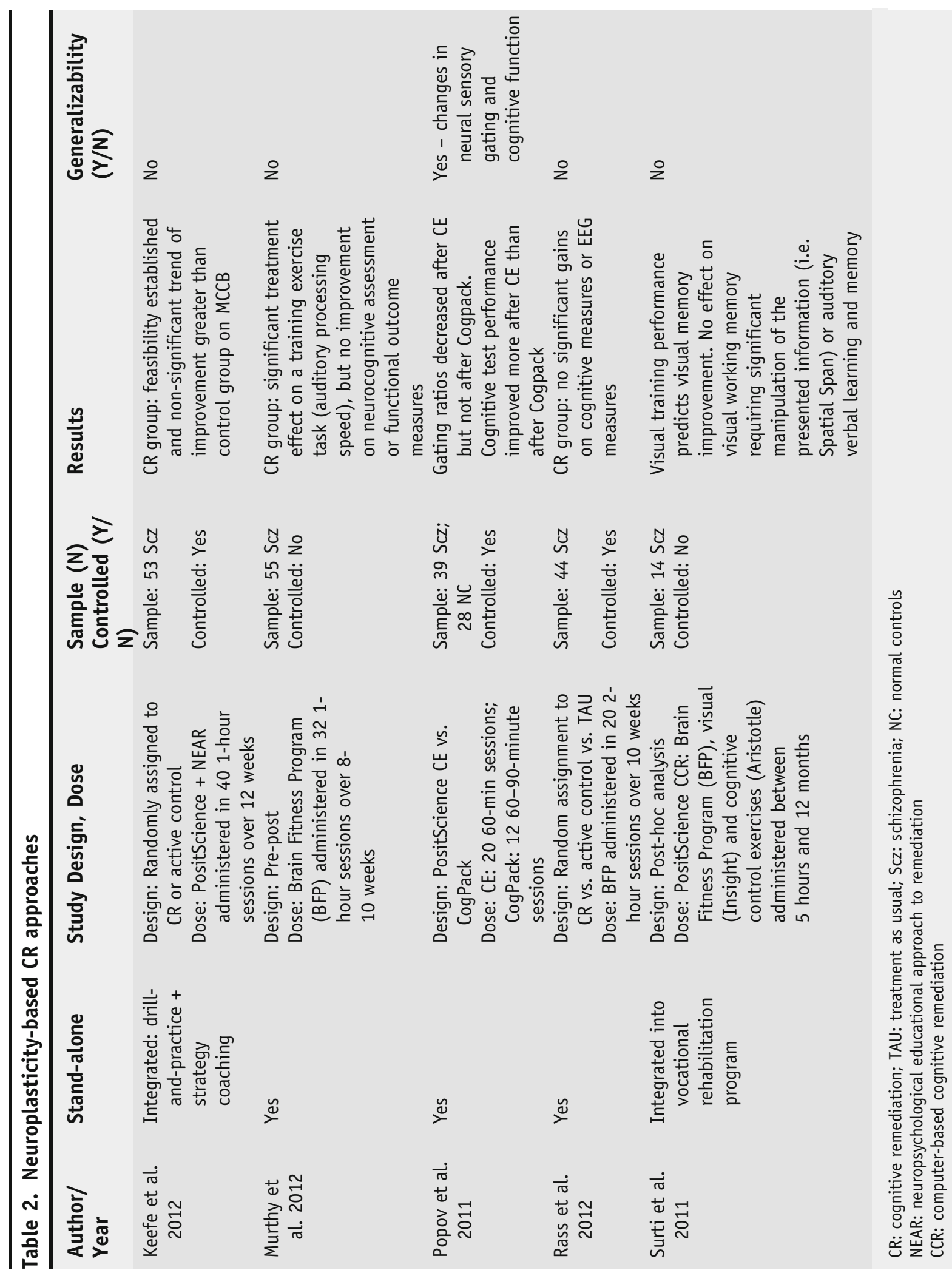




\section{Conclusions}

month follow-up. Thus, the limited data available for this review suggest neuroplasticity-based interventions likely impact functional brain changes associated with basic perceptual/cognitive processes.

Finally, similar to higher-level intervention studies, neuroplasticitybased approaches have been implemented in two formats: drill and practice, and drill and practice integrated with another psychosocial treatment. One of the five studies combined neuroplasticity-based CR with NEAR strategy-coaching "bridging" groups and another study used adjunctive vocational rehabilitation. Neither found significant treatment gains in cognition or functional outcomes. Of the three studies that did not include adjunctive psychiatric rehabilitation, two studies observed gains on tasks similar to those used in training, and one study found no improvements in the treatment group. Thus, the data from the five studies included in this review do not indicate an observable benefit of adjunctive treatment.

Taken together, studies from the past 3 years shed additional light on some of the salient issues in the literature on CR in schizophrenia. Regarding the first question of whether apparent treatment benefits generalize to other neurocognitive domains and functional outcome, the majority of recent studies using higher-level approaches reported generalizability of cognitive gains to other domains of cognition and to functional outcomes. In contrast, few of the recent neuroplasticity-based interventions found evidence of generalization to abilities apart from those directly related to the training exercises. The multi-site trial can be viewed as partial support for neuroplasticity-based interventions and cognitive change because the results were significant at the midpoint but not the endpoint. More studies are needed to fully understand the generalizability of neuroplasticity-based CR. In contrast, the evidence of generalizability has grown stronger for higher-level CR interventions.

Regarding the second question about impact on brain functioning, there is a small amount of evidence for higher-level approaches and mixed findings for neuroplasticity-based CR. Changes at the level of structural or functional imaging, or MEG and EEG, will be more meaningful if they are observed in concert with cognitive or functional gains. Regarding the third question of whether these approaches are best administered as stand-alone vs. integrated treatments, the jury is still out. Conceptually, it makes sense that gains from CR would augment gains from skills training or vocational rehabilitation; however, the current review suggests patients benefit with or without adjunctive psychosocial rehabilitation, at least on the functional measures included in the present studies. The study by Bowie and colleagues [12] is the one exception among recent studies to this conclusion: they found that persons receiving CR only showed improvements in neurocognition and those in the combined $\mathrm{CR}+$ skills-training showed gains in social and functional competence, real-world community activities, and work skills. These findings are important and suggest a highly specific effect of CR alone versus CR + 
adjunctive training, but it is the only higher-level study in our update with a controlled experimental design to specifically make this comparison. Thus, there is currently no clear evidence that neuroplasticity-based CR provides more benefits than traditional higher-level approaches. Only one study has directly compared these approaches by randomly assigning 39 schizophrenia patients to either 20 60-minute sessions of BrainFitness CE or 12 60-90-minute sessions of CogPack [32]. In this study, betweengroup comparisons were mixed and both treatment groups had significant improvements in cognition, but the neuroplasticity-based group had larger effect sizes for cognitive gains.

The current update reveals notable recent trends and advances in CR research, such as the integration of adjunctive psychiatric rehabilitation and increased utilization of neuroscience assessment techniques. There are a few important areas where future research should be directed. First, additional studies are needed to examine minimally necessary and sufficient dosage and frequency for treatment gains. The dosage requirements will likely depend on individual characteristics including level of baseline deficits. Second, the technique of adjusting difficulty level to match ability level has been supported in reviews, but never empirically compared to a general format with preset difficulty levels. To date, only one intervention (Recos) applied CR to specific domains dependent on baseline deficits of an individual patient. A third issue is that it is difficult to compare results across studies since a wide variety of assessment methods have been used. Future research would be benefited by the use of standardized assessments and well-normed neurocognitive batteries. The MATRICS Consensus Cognitive Battery (MCCB) [40] is accepted for use in clinical trials and facilitates comparisons across interventions and across samples. It would help to develop similar standardized methodologies for domains of community functioning and that was the goal of the NIMH VALERO study [41]. Finally, there is some hope that cognitive remediation might help with the large problem of medication adherence in schizophrenia. However, the literature on cognition and adherence is inconsistent, and some of the larger studies paradoxically find that better cognition is associated with poorer medication adherence $[42,43]$. Hence, medication adherence will be an elusive target for cognitive remediation, as its causes are complex. One related question is whether patients with better medication adherence demonstrated stronger improvements with $\mathrm{CR}$, and studies are currently underway to address that issue.

Next steps for CR include clinical implementation and dissemination. Several studies support the implementation of CR in real-world clinical settings $[17,20,21,44]$, although there remains uncertainty about the specific parameters of implementation (e.g., duration, approach, dosage, etc.). As stated above, computerized programs can ease the burden of dissemination in that they require less staff training, and enhance standardization of procedures. The current CR literature suggests CR interventions are efficacious for people with schizophrenia. Further information regarding dosage, individually tailored treatments, and standardization of assessments will enable the field to move forward with effectiveness studies to establish benefits across diverse settings and patient groups. In looking forward to future directions of CR research, 
current research trends suggest that combining CR with new approaches, such as social cognitive remediation and cognitive enhancing medication, will be increasingly important. These are active research areas with several trials currently underway.

\section{Compliance with Ethics Guidelines}

\section{Conflict of Interest}

Carol Jahshan, L. Felice Reddy and William P. Horan declare no conflict of interest. Michael F. Green reports previous work as a consultant for Brain Plasticity, which produces cognitive remediation software, outside of the submitted work.

Human and Animal Rights and Informed Consent

This article does not contain any studies with animal subjects performed by any of the authors.

\section{References and Recommended Reading}

Papers of particular interest, published recently, have been highlighted as:

- Of importance

1. Green MF et al. Neurocognitive deficits and functional outcome in schizophrenia: Are we measuring the "right stuff"? Schizophr Bull. 2000;26:119-36.

2. Green MF, Kern RS, Heaton RK. Longitudinal studies of cognition and functional outcome in schizophrenia: implications for MATRICS. Schizophr Res. 2004;72:41-51.

3. Bilder RM. Neuropsychology and neurophysiology in schizophrenia. Curr Opin Psychiatr. 1996;9(1):5762.

4. Green MF et al. From perception to functional outcome in schizophrenia: modeling the role of ability and motivation. Arch Gen Psychiatr. 2012;69(12):1216-24.

5. Hoff AL, Kremen WS. Neuropsychology in schizophrenia: an update. Curr Opin Psychiatr. 2003;16(2):149-55.

6. Medalia A. Cognitive remediation for psychotic and affective disorders. Can J Psychiatr Rev Canad Psychiatr. 2013;58(6):309-10.

In this introduction to review issue, Medalia asserts that neuroplasticity is associated with both computerized interventions and with rehabilitation-oriented interactive approaches. Further, integrative approaches to CR are effective in dissemination trials. The debate is currently between medical model "dosed" device-reliant approaches and recovery model interactive and applied approaches.

7. Buonomano DV, Merzenich MM. Cortical plasticity: from synapses to maps. Annu Rev Neurosci. 1998;21:149-86.
8. Genevsky A et al. Cognitive training in schizophrenia: a neuroscience-based approach. Dialogues Clin Neurosci. 2010;12(3):416-21.

9. Wykes $\mathrm{T}$ et al. A meta-analysis of cognitive remediation for schizophrenia: methodology and effect sizes. Am J Psychiatr. 2011;168(5):472-85.

Meta-analysis of 40 studies, including 2,104 participants, to compare treatment elements with cognitive outcome and durability of effects.

10. Deppen $P$ et al. Cognitive remediation program for individuals living with schizophrenia (Recos): preliminary results. L'Encéphale. 2011;37(4):314-21.

11. Franck $\mathrm{N}$ et al. Specific vs general cognitive remediation for executive functioning in schizophrenia: a multicenter randomized trial. Schizophr Res. 2013;147(1):68-74.

12. Bowie CR et al. Combined cognitive remediation and functional skills training for schizophrenia: effects on cognition, functional competence, and real-world behavior. Am J Psychiatr. 2012;169(7):710-8.

13. Bucci $P$ et al. Neurocognitive individualized training versus social skills individualized training: a randomized trial in patients with schizophrenia. Schizophr Res. 2013;150(1):69-75.

14. Cella $\mathrm{M}$, et al. Identifying Cognitive Remediation Change Through Computational Modelling-Effects on Reinforcement Learning in Schizophrenia. Schizophr Bull. 2013. doi:10.1093/schbul/sbt152.

15. Garrido $\mathrm{G}$ et al. Computer-assisted cognitive remediation therapy: cognition, self-esteem and quality of 
life in schizophrenia. Schizophr Res. 2013;150(23):563-9.

16. Lee WK. Effectiveness of computerized cognitive rehabilitation training on symptomatological, neuropsychological and work function in patients with schizophrenia. Asia Pac Psychiatr. 2013;5(2):90-100

17. Lindenmayer JP et al. Improving social cognition in schizophrenia: a pilot intervention combining computerized social cognition training with cognitive remediation. Schizophr Bull. 2013;39(3):507-17.

18. Royer A et al. Is there any impact of cognitive remediation on an ecological test in schizophrenia? Cogn Neuropsychiatr. 2012;17(1):19-35.

19. Trapp W et al. Cognitive remediation improves cognition and good cognitive performance increases time to relapse-results of a 5 year catamnestic study in schizophrenia patients. BMC Psychiatr. 2013;13:184.

20. Tan BL, King R. The effects of cognitive remediation on functional outcomes among people with schizophrenia: a randomised controlled study. Aust New Zeal J Psychiatr. 2013;47(11):1068-80.

21. Vita A et al. Effectiveness of different modalities of cognitive remediation on symptomatological, neuropsychological, and functional outcome domains in schizophrenia: a prospective study in a real-world setting. Schizophr Res. 2011;133(1-3):223-31.

22. d'Amato $\mathrm{T}$ et al. A randomized, controlled trial of computer-assisted cognitive remediation for schizophrenia. Schizophr Res. 2011;125(2-3):284-90.

23. Gharaeipour M, Scott BJ. Effects of cognitive remediation on neurocognitive functions and psychiatric symptoms in schizophrenia inpatients. Schizophr Res. 2012;142(1-3):165-70.

24. Bor J et al. How can cognitive remediation therapy modulate brain activations in schizophrenia? An fMRI study. Psychiatr Res. 2011;192(3):160-6.

25. Penades R et al. Brain effects of cognitive remediation therapy in schizophrenia: a structural and functional neuroimaging study. Biol Psychiatr. 2013;73(10):1015-23.

26. Eack SM et al. Neuroprotective effects of cognitive enhancement therapy against gray matter loss in early schizophrenia: results from a 2-year randomized controlled trial. Arch Gen Psychiatr. 2010;67(7):674-82.

27. Haut KM, Lim KO, MacDonald 3rd A. Prefrontal cortical changes following cognitive training in patients with chronic schizophrenia: effects of practice, generalization, and specificity.

Neuropsychopharmacology. 2010;35(9):1850-9.

28. McGurk SR et al. A meta-analysis of cognitive remediation in schizophrenia. Am J Psychiatr. 2007;164(12):1791-802.

29. Keefe RS et al. Feasibility and pilot efficacy results from the multisite Cognitive Remediation in the Schizophrenia Trials Network (CRSTN) randomized controlled trial. J Clin Psychiatr. 2012;73(7):1016-22.
30. Murthy NV et al. Computerized cognitive remediation training for schizophrenia: an open label, multisite, multinational methodology study. Schizophr Res. 2012;139(1-3):87-91.

The first large, multi-site study of CR in schizophrenia. The study implemented "dosed" computerized CR and failed to replicate the findings of a previous single-site study of computer-based CR.

31. Surti TS et al. Successful computer-based visual training specifically predicts visual memory enhancement over verbal memory improvement in schizophrenia. Schizophr Res. 2011;132(2-3):131-4.

32. Popov $\mathrm{T}$ et al. Specific cognitive training normalizes auditory sensory gating in schizophrenia: a randomized trial. Biol Psychiatr. 2011;69(5):465-71.

33. Bell MD, et al. Benefits of Cognitive Remediation and Supported Employment for Schizophrenia Patients with Poor Community Functioning. Psychiatr Serv 2014. doi:10.1176/appi.ps.201200505.

34. Fisher $\mathrm{M}$ et al. Using neuroplasticity-based auditory training to improve verbal memory in schizophrenia. Am J Psychiatr. 2009;166(7):805-11.

35. Popov $\mathrm{T}$ et al. Adjusting brain dynamics in schizophrenia by means of perceptual and cognitive training. PloS One. 2012;7(7):e39051.

36. Rass $\mathrm{O}$ et al. Computer-assisted cognitive remediation for schizophrenia: a randomized single-blind pilot study. Schizophr Res. 2012;139(1-3):92-8.

37. Adcock RA et al. When top-down meets bottom-up: auditory training enhances verbal memory in schizophrenia. Schizophr Bull. 2009;35(6):1132-41.

38. Dale CL et al. Timing is everything: neural response dynamics during syllable processing and its relation to higher-order cognition in schizophrenia and healthy comparison subjects. Int J Psychophysiol. 2010;75(2):183-93.

39. Fisher $\mathrm{M}$ et al. Neuroplasticity-based cognitive training in schizophrenia: an interim report on the effects 6 months later. Schizophr Bull. 2010;36(4):869-79.

40. Nuechterlein KH, Green MF. MATRICS Consensus Cognitive Battery. Los Angeles: MATRICS Assessment, Inc; 2006.

41. Harvey PD et al. Validating the Measurement of RealWorld Functional Outcomes: Phase I Results of the VALERO Study. Am J Psychiatr. 2011;168(11):1195-201.

42. Jónsdóttir $\mathrm{H}$ et al. Predictors of medication adherence in patients with schizophrenia and bipolar disorder. Acta Psychiatr Scand. 2013;127:23-33.

43. Perkins DO et al. Predictors of treatment discontinuation and medication nonadherence in patients recovering from a first episode of schizophrenia, schizophreniform disorder, or schizoaffective disorder: a randomized, double-blind, flexible-dose, multicenter study. J Clin Psychiatr. 2008;69(1):106-13.

44. Eack SM. Cognitive remediation: a new generation of psychosocial interventions for people with schizophrenia. Social Work. 2012;57(3):235-46. 